\title{
Immunohistochemical study of serotonin in lesions of chronic eczema and psoriasis
}

\author{
Issam Msakni, Raoueh Hedhli, Karima Tlili, Nada Mansouri, Faten Gargouri, \\ Basma Laabidi
}

Departement of Pathology, Military Hospital of Tunis, Tunisia

Corresponding author: Dr. Karima Tlili, E-mail: tlili.karima@yahoo.fr

\begin{abstract}
Background: Psoriasis and chronic eczema are common chronic inflammatory skin diseases. Their prevalence is variable. Several factors participate in their pathogenesis such as serotonin. Our objective was to study the role of serotonin in the pathogenesis of chronic eczema and psoriasis. We studied the distribution and expression of serotonin in these lesions in comparison with skin specimens from control subjects. Methods: It was a retrospective and monocentric study of 27 patients, collected at the Department of Pathology between 2008 and 2016. They were thirteen cases of chronic eczema and fourteen cases of psoriasis. Six cases of normal skin were included for negative control. The demographic, pathological and immunohistochemical data were available for all patients. Results: The age of patients ranged from 6 to 84 years old. Most patients were males with a sex ratio of 1,75. Mean age was 45 years for patients with eczema and 44 years for those with psoriasis. Serotonin was expressed in 6 cases of eczema and in 2 cases of psoriasis. Conclusion: Serotonin participates in the pathogenesis of chronic eczema and psoriasis. A targeted anti-serotonin therapy may be beneficial in lesions expressing serotonin.
\end{abstract}

Key words: Eczema; Psoriasis; Serotonin

\section{INTRODUCTION}

Psoriasis and chronic eczema are common, chronic inflammatory skin diseases.

The most common form of chronic eczema is atopic dermatitis. It is the most common chronic inflammatory skin disease that often begins in childhood [1]. Its prevalence varies by age and country. It is rising dramatically, especially in developed countries, and it now affects $10-30 \%$ of children and $1-10 \%$ of adults [2].

Psoriasis is an heterogeneous immune-mediated chronic inflammatory disease, which can affect the skin, nails and joints [3]. Its prevalence varies between 0.05 and $3.7 \%$ depending on ethnicity and geographic location [4].

Several factors participate in the pathogenesis and pathophysiology of chronic inflammatory skin disease such as inflammatory mediators. Several study suggested that 5-hydroxytryptamine (5-HT serotonin,) may participate in the eczematous and psoriatic process $[5,6]$.

5-HT is a multi-functional bioamine with important signaling roles in a range of physiological pathway [7]. It is mostly synthesized in enterocromaffin cells within the gastrointestinal mucosa. A small amount of 5-HT is synthesized by serotonergic neurons within the central nervous system, pancreatic islets, mammary glands and adipose tissue [7].

Our objective was to study the role of serotonin in the pathogenesis of chronic eczema and psoriasis. Using immunohistochemical techniques, we studied the distribution and expression of serotonin in these lesions in comparison with skin specimens from control subjects.

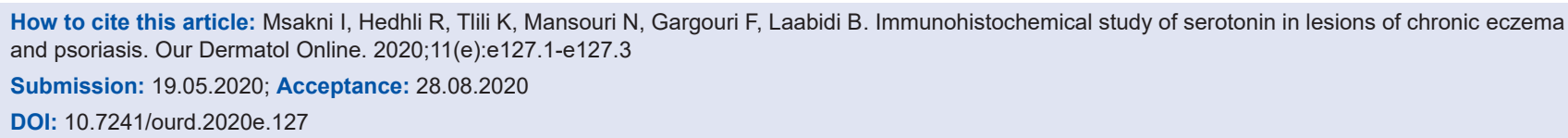




\section{METHODS}

It was a retrospective and monocentric study of 27 cases, collected at the Department of Pathology between 2008 and 2016. They were thirteen cases of chronic eczema and fourteen cases of psoriasis. Six cases of normal skin were included for negative control.

All cases studied were biopsy specimens without other skin lesions.

Clinical data including sex, age, were collected from clinical records.

Pathologic data were collected from histopathological reports.

Immunohistochemical study used the monoclonal anti-serotonin antibody (DAKA, clone 5HT-H209).

\section{RESULTS}

The age of patients ranged from 6 to 84 years old. The age group most presented was between 40 and 60 years old with $36 \%$ of cases. Most patients were males with a sex ratio of 1,75 .

Mean age was 45 years for patients with eczema and 44 years for those with psoriasis.

Immunolabeling was absent in all cases of normal skin. Serotonin was expressed in 6 cases of eczema. The immunolabeling was observed in the spinous and basal cells as well as the hair follicles. Serotonin was expressed in 2 cases of psoriasis. The immunolabeling was observed in the horn, the spinous and the granular cells.

\section{DISCUSSION}

Several study suggested that inflammatory mediators such as serotonin may participate in the pathogenesis of skin disease like eczema and psoriasis.

A series published by Huang J et al. [5] studied 25 cases of various types of chronic eczema and a control group consisted of 10 people. It showed that specimens from all 10 control subjects had negative expressions of serotonin. For patients with chronic eczema, expression of serotonin in the prickle cell cytoplasm was moderately positive in 20 cases, and weakly positive in five cases. The expression in sweat gland cytoplasm and in sebaceous gland was moderately positive in 22 cases and weakly positive in three cases. The expression in hair follicle cytoplasm was moderately positive in 21 cases and weakly positive in four cases.

Several study [8-11] showed that a selective serotonin reuptake inhibitor (SSRI), such as paroxetine, fluvoxamine and fluoxetine are effective in the treatment of atopic dermatitis-like skin lesions in mice. These effects are mediated by regulating proinflammatory cytokine.

A series published by Huang J et al. [6] studied 35 cases of psoriasis and a control group consisted of 10 people. Thirty-one patients had vulgaris-type psoriasis, and four patients had the pustular type. Twenty-nine patients had progressive stage and six patients had static disease. It showed that specimens from all 10 control subjects had negative expressions of serotonin. The expression of serotonin in patients whose psoriasis was in the progressive stages, prickle cells was extensively positive. Greater positive expression was evident in the sweat gland cells, sebaceous gland cells, and hair roots. The expression of serotonin in patients, whose psoriasis was in the static stage, was less evident than in those in the progressive stage. No difference was detected between the vulgaris type and the pustular type.

Psoriasis was reported to be a stress-induced chronic inflammation [12]. Stress and stress-related hormones has been well documented to be connected to the serotonergic system.

A study published by Thorslund K et al. [13] studied 20 biopsies from involved and non-involved skin from the back of 20 patients with chronic plaque psoriasis. An immunohistochemically study using a monoclonal antibody to serotonin transporter protein (SERT) was done. Severity index of psoriasis (PASI) was determined. Levels of depression and chronic stress were measured using Beck's Depression Inventory (BDI) and the salivary cortisol test, respectively. The numbers of SERT-positive cells in the epidermis of involved psoriatic skin was positively correlated with PASI. It was negatively correlated with salivary cortisol ratio levels, indicating a correlation between SERT expression and chronic stress.

Another study published by Peters EMJ et al. [14] also showed the correlation between stress, serotonin and psoriasis and the benefit of this interaction to be exploited therapeutically both through pharmacological 
approaches and psychotherapy to improve the treatment of psoriasis.

\section{CONCLUSION}

Our study demonstrated the expression of serotonin in chronic eczema and psoriasis while it is absent in normal skin. This neurotransmitter participates in the pathogenesis of these lesions via its role in the immune response and the inflammatory process in association with other mediators. An immunohistochemical study is recommended in these cases to select patients for whome targeted anti-serotonin and psychotherapy may be beneficial.

\section{Statement of Human and Animal Rights}

All procedures followed were in accordance with the ethical standards of the responsible committee on human experimentation (institutional and national) and with the Helsinki Declaration of 1975, as revised in 2008 .

\section{Statement of Informed Consent}

Informed consent was obtained from all patients for being included in the study.

\section{REFERENCES}

1. Kaufman BP, Guttman-Yassky E, Alexi AF. Atopic dermatitis in diverse racial and ethnic groups - variations in epidemiology, genetics, clinical presentation, and treatment. Exp Dermatol. 2018;27:340-57.

2. Li Y, Chen L, Du Y, Huang D, Han H, Dong Z. Fluoxetine ameliorates atopic dermatitis-like skin lesions in balb/c mice through reducing psychological stress and inflammatory response. Front Pharmacol. 2016;7:318.

3. Weigle N, McBane S. Psoriasis. Am Fam Physician. 2013;87:626-33.

4. Parisi R, Symmons DP, Griffiths CE, Ashcroft DM. Global epidemiology of psoriasis: a systematic review of incidence and prevalence. J Invest. Dermatol. 2013;133:377-85.

5. Huang J, Li G, Xiang J, Yin D, Chi R. Immunohistochemical study of serotonin in lesions of chronic eczema. Int J Dermatol. 2004;43:723-6.

6. Kelati A, Meziane M, Jaafari M, Mernissi FZ. The impact of psoriasis on the lifequality: a cohort of 140 Moroccan patients. Our Dermatol Online. 2016;7:10-6.

7. Martin AM, Young RL, Leong L, Rogers GB, Spencer NJ, Jessup CF, et al.. The diverse metabolic roles of peripheral serotonin. Endocrinology. 2017;158:1049-63.

8. Blessmann Weber M, Kanaan Recuero J, Saraiva Almeida C. Use of psychiatric drugs in dermatology. An Bras Dermatol. 2020;95:133-43.

9. Rachel SG, Juan MG, Gil Y. Practical approach for the Diagnosis and treatement of chronic Prurits. J Nurpra. 2020;05:2.

10. Song J, Xian D, Yang L, Xiong X, Lai R, Zhong J. Pruritus: Progress toward Pathogenesis and Treatment. Biomed Res Int. 2018;2018:9625936.

11. Li Y, Chen L, Du Y, Huang D, Han H, Dong Z. Fluoxetine ameliorates atopic dermatitis-like skin lesions in BALB/c mice through reducing psychological stress and inflammatory response. Front Pharmacol. 2016;7:31 .

12. Ronpirin $\mathrm{C}$ and Tencomnao T. Psoriasis: A review of the role of serotonergic system. Afr J Biotechnol. 2010;9:1528-34.

13. Thorslund K, Amatya B, Dufva AE and Nordlind K. The expression of serotonin transporter protein correlates with the severity of psoriasis and chronic stress. Arch Dermatol Res. 2013;305:99-104.

14. Peters EMJ, Sabat R, Philipp S, Helleiner E, Kruse J and Sterry W. Neuroimmunological links in dermatology: psoriasis as a model disease in stress research. Expert Rev Dermatol. 2012;7:367-75.

Copyright by Issam Msakni, et al. This is an open access article distributed under the terms of the Creative Commons Attribution License, which permits unrestricted use, distribution, and reproduction in any medium, provided the original author and source are credited.

Source of Support: Nil, Conflict of Interest: None declared. 